\title{
AN OPTIMAL PROMOTION COST CONTROL MODEL FOR A MARKOVIAN MANPOWER SYSTEM
}

A. U. UDOM AND P. I. UCHE

(Received 6 November 2008; Revision Accepted 4 March 2009)

\section{ABSTRACT}

A theory concerning the existence of an optimal promotion control strategy for controlling a Markovian manpower system is developed. Control interventions are made at controller's specific time point with cost as an optimality performance criterion. A dynamic programming technique is adopted for the optimal solution and the model is applied to a hypothetical three-grade manpower system.

KEYWORDS: Cost, Existence, Optimal Control, Markovian, Manpower System

\section{INTRODUCTION}

Many models have been developed for both homogenous and non-homogenous manpower systems. These models range from supply and demand forecasting models to control models within the Markov, semi-Markov and renewal frameworks. Some of these models are transition models and are concerned with the effect of constant transition probabilities on changing class structure. These models enable us to see how the aggregate behaviour of the system depends on the propensity to move members. See for example, Gani (1963), Young and Almond (1961). Whereas the control models have two specific aspects, namely: attainability which is concerned with whether or not a goal can be reached and if so by what means and maintainability which deals on how to remain on a given desired structure once it has been attained, for example, Nwaigwe (2008).

One of the main purposes of manpower modeling is to analyze the effect of stocks and flows on the size and relative structure of an organization. In recent times, a number of authors have developed the theory of transition models in manpower studies. Among the various models under consideration, there exists a specific group of models that can generally be referred to as control models for Markovian manpower system. See for example Mehlmann (1980) and Davies (1982).

Certain level of control is required in manpower modeling at various times and for various reasons. Uche (1984) develops a promotion control model for a Markovian system by solving a control equation for the promotion rates. Kareem (2005), identifies various strategies for effective manpower planning through mathematical models based on multiple activity process chart, queuing system approach and linear programming models. With these models, optimal number of manpower to be employed for profitable operations can be obtained. Fry, et al (2003), examine the problem of determining the optimal staffing level for a fire department by using an inventory quantitative model that allows for stochastic temporary absences, permanent wastage and limited hiring opportunities to derive implementable optimal polices for both the continuous and discrete cases. The work provides numerical results comparing the optimal solution to several heuristic staffing polices used in practice which shows that the optimal policy can provide significant savings. They also perform sensitivity analyses to see the effect of parameter change on the model.

A critical and most difficult aspect of manpower control is the specification of the best control strategy in the event of having two or more control strategies capable of controlling a system. Bartholomew (1982) investigates control strategies (adaptive and fixed) with the main aim 
of bringing the relative personnel structure as close to a goal as possible. This is done by introducing some measure of distance between the existing structure and the desired structure using mathematical programming technique. However, these strategies give no insight to the cost required to move the personnel structure to a desired or near desired structure. Few questions arise: What would be the decision a planner takes so as to optimize a given cost function arising from flows in the system? Suppose that there are two or more promotion strategies that can transfer the system from a given structure to the desired structure, which of these strategies would be the best?

In view of the above questions our aim in this work is to develop an optimal promotion control model for a Markovian manpower system, where cost is used as an optimality performance criterion for distinguishing promotion strategies. Our interest is on systems where transitions are governed by Markov processes and the cost structure of the process is described by a matrix that represents the cost associated with movement from one state to another.

Although many approaches to manpower capacity planning exist, the practicality of such approaches depends on how easily such models can be implemented and the ease with which the models can be tailored to the situation at hand. Hence we propose the optimal cost control model. The optimal cost model is a special kind of control known as control by intervention or impulse control. This concerns a situation in which a manpower system runs uncontrolled until the decision maker decides to intervene moving the process instantaneously to some new point in the state space from which its motion continues until a new intervention is made, and so on. Here we assume that it is never positively advantageous to move from state $\mathrm{i}$ to $\mathrm{j}$ via a third state $\mathrm{k}$. The effect of this assumption is that it will never be optimal to do anything other than make a sequence of isolated interventions.

\section{MARKOVIAN MANPOWER SYSTEM}

Consider a manpower system whose members are divided into $k$ categories. Let $n_{i}(T)$ denote the number of people in category $i$ at time $T(T=0,1,2, \ldots), N(T)=\sum_{i} n_{i}(T)$ the total number in the system and $R(T)$ the number of recruits to the system at time $T$. A member of category $\mathrm{i}$ moves to category $\mathrm{j}$ with probability $P_{i j}$; where $\sum_{j}^{k} P_{i j}<1$. Because transition of members out of the system is allowed; we have $P_{i, k+1}$ to be the probability of a member in category i moving out of the system such that $\sum_{j} P_{i j}+P_{i, k+1}=1$. The total number of recruits $\mathrm{R}(\mathrm{T})$ is distributed to the k-categories according to the proportion $P_{0 i}$ with $\sum_{i} P_{0 i}=1$ Using the above notations, the system can be described with the following recursive relation:

$n_{i}(T+1)=\sum_{i}^{k} P_{i j} n(T)+R(T+1) P_{0 j}$

or in matrix form

$n(T+1)=n(T) P+R(T+1) P_{0}$

Control of the above system can be exercised through recruitment or promotion. When the only flow subject to change by management for the purpose of control is promotion flow, it is referred to as Promotion control.

Suppose that the relative grade size is to be controlled, this means that we are to find a promotion strategy $\mathrm{P}$ satisfying the equation $q=q P+q w^{\prime} r$. The problem here is that such a $\mathrm{P}$ is not unique. See for example, Uche (1984). Ossai (2008) also identifies this problem in a departmentalized control model. To distinguish the admissible $P$ for optimality we introduce cost function to the admissible $P$ as an optimality criterion and this is shown in the following section. 


\section{OPTIMAL PROMOTION COST CONTROL MODEL}

Consider a Markovian manpower system observed at time $k=0,1,2, \ldots s$ to be in any of the states $N_{k}$ and after observing the state of the system, an action must be taken. Let $a_{k}$ be the action taken where $a_{k} \in \pi$ is a set of admissible promotion control strategies that can move $N_{k}$ to $\mathrm{N}_{\mathrm{k}}{ }^{*}$, where $\mathrm{N}_{\mathrm{k}}{ }^{*}$ is a desired structure we want to maintain. Let $C\left(N_{k}, a_{k}\right)$ be the cost incurred in moving the system from $N_{k}$ to $N_{k}^{*}$ with $\mathrm{a}_{\mathrm{k}}$ as the action taken. Assume that the cost $\mathrm{C}\left(\mathrm{N}_{\mathrm{k}}, \mathrm{a}_{\mathrm{k}}\right)$ is bounded such that $\left|\mathrm{C}\left(\mathrm{N}_{\mathrm{k}}, \mathrm{a}_{\mathrm{k}}\right)\right|<\mathrm{M}$

Let $V_{\pi}\left(N_{k}, a_{k}\right)=\mathrm{C}\left(\mathrm{N}_{\mathrm{k}}, \mathrm{a}_{\mathrm{k}}\right)+\sum_{j} P_{i j}\left(a_{k}\right) V_{\pi}\left(N_{k}\right)$.

be the expected total cost incurred when $\pi$ is the promotion strategy chosen and the initial state is $\mathrm{N}_{\mathrm{k}}$. The problem now is to minimize $V_{\pi}\left(N_{k}, a_{k}\right)=\mathrm{C}\left(\mathrm{N}_{\mathrm{k}}, \mathrm{a}_{\mathrm{k}}\right)+\sum_{j} P_{i j}\left(a_{k}\right) V_{\pi}\left(N_{k}\right)$ where $V_{\pi}\left(N_{k}\right)$ any other is cost incurred in the course of attaining the desired structure.

Let $V\left(N_{k}^{*}, a_{k}\right)=\inf _{\pi} V_{\pi}\left(N_{k}, a_{k}\right)$, then $\pi^{*}$ is an optimal promotion strategy if

$V_{\pi^{*}}\left(N_{k}, a_{k}\right)=V\left(N_{k}^{*}, a_{k}\right)$

In the following demonstration, it is shown that $V\left(N_{k}^{*}, a_{k}\right)$ is the unique minimum of $V_{\pi}\left(N_{k}, a_{k}\right)$, that is

$$
V_{\pi^{*}}\left(N_{k}, a_{k}\right)=\operatorname{Min}_{a_{k}}\left\{\mathrm{C}\left(\mathrm{N}_{\mathrm{k}}, \mathrm{a}_{\mathrm{k}}\right)+\sum_{j} P_{i j}\left(a_{k}\right) V_{\pi}\left(N_{k}\right)\right\} \text {. }
$$

Proof: Let $\pi$ be an arbitrary promotion policy and suppose that $\pi$ chooses action $a_{k}$ at time $k$ with probability $P_{a_{k}}$; Then

$$
V_{\pi}\left(N_{k}, a_{k}\right)=\sum_{a_{k}} \mathrm{P}_{\mathrm{a}_{\mathrm{k}}}\left\{\mathrm{C}\left(\mathrm{N}_{\mathrm{k}}, \mathrm{a}_{\mathrm{k}}\right)+\sum_{j} P_{i j}\left(a_{k}\right) V_{\pi}\left(N_{k}\right)\right\} \text {. }
$$

where $V_{\pi}\left(N_{k},\right)$ represents the expected cost incurred from time $k=1$ onward in the course of attaining the desired structure. Then it follows that

$V_{\pi}\left(N_{k}, a_{k}\right) \geq V_{\pi^{*}}\left(N_{k}, a_{k}\right)$

Hence

$$
\begin{aligned}
V_{\pi}\left(N_{k}, a_{k}\right) \geq \sum_{a_{k}} \mathrm{P}_{\mathrm{a}_{\mathrm{k}}}\left\{\mathrm{C}\left(\mathrm{N}_{\mathrm{k}}, \mathrm{a}_{\mathrm{k}}\right)+\sum_{j} P_{i j}\left(a_{k}\right) V_{\pi^{*}}\left(N_{k}\right)\right\} \\
\geq \sum_{a_{k}} \mathrm{P}_{\mathrm{a}_{\mathrm{k}}} \operatorname{Min}_{a}\left\{\mathrm{C}\left(\mathrm{N}_{\mathrm{k}}, \mathrm{a}_{\mathrm{k}}\right)+\sum_{j} P_{i j}\left(a_{k}\right) V_{\pi^{*}}\left(N_{\mathrm{k}}\right)\right\} . \\
=\operatorname{Min}_{a_{k}}\left\{\mathrm{C}\left(\mathrm{N}_{\mathrm{k}}, \mathrm{a}_{\mathrm{k}}\right)+\sum_{j} P_{i j}\left(a_{k}\right) V_{\pi^{*}}\left(N_{k}\right)\right\}
\end{aligned}
$$

Since $\pi$ is arbitrary, it implies that

$$
V_{\pi^{*}}\left(N_{k}, a_{k}\right) \leq \operatorname{Min}_{a}\left\{\mathrm{C}\left(\mathrm{N}_{\mathrm{k}}, \mathrm{a}_{\mathrm{k}}\right)+\sum_{j} P_{i j}\left(a_{k}\right) V_{\pi^{*}}\left(N_{k}\right)\right\} \ldots
$$

On the other hand let $m$ be the policy that choose $a_{0}$ at time $k=0$; such that

$$
C\left(N_{k}, a_{0}\right)+\sum_{j} P_{i j}\left(a_{0}\right) V_{\pi}\left(N_{k}\right)=\operatorname{Min}_{a k}\left\{\mathrm{C}\left(\mathrm{N}_{\mathrm{k}}, \mathrm{a}_{\mathrm{k}}\right)+\sum_{j} P_{i j}\left(a_{k}\right) V_{\pi}\left(N_{k}\right)\right\} .
$$

Then

$$
\begin{array}{r}
V_{\pi}\left(N_{k}, a_{k}\right)=C\left(N_{k}, a_{0}\right)+\sum_{j} P_{i j}\left(a_{0}\right) V_{\pi}\left(N_{k}\right) \\
\geq C\left(N_{k}, a_{0}\right)+\sum_{l} P_{i j}\left(a_{0}\right) V_{\pi^{*}}\left(N_{k}\right) \ldots
\end{array}
$$


since $V_{\pi^{*}}\left(N_{k}, a_{k}\right) \leq V_{\pi}\left(N_{k}, a_{k}\right)$.

This implies that

$$
V_{\pi^{*}}\left(N_{k}, a_{k}\right) \leq C\left(N_{k}, a_{0}\right)+\sum_{j} P_{i j}\left(a_{0}\right) V\left(N_{k}\right)
$$

Hence from 7 we obtain that

$$
V_{\pi^{*}}\left(N_{k}, a_{k}\right) \leq \operatorname{Min}_{a_{k}}\left[C\left(N_{k}, a_{k}\right)+\sum_{j} P_{i j}\left(a_{k}\right) V\left(N_{k}\right)\right]
$$

Thus $V_{\pi^{*}}\left(N_{k}, a_{k}\right)$ is the optimal value of $V_{\pi}\left(N_{k}, a_{k}\right)$.

\section{METHOD OF SOLUTION FOR INFINITE HORIZON}

We present here a method of obtaining the optimal promotion control strategy using the dynamic programming technique. Infinite horizon here means that the transition probabilities and their cost functions are assumed to be stationary. Consider a situation where there are two or more promotion control strategies and $a_{k}$ is chosen with $P_{a_{k}}$ and $C\left(N_{k}, a_{k}\right)$ as the transition and cost matrices associated with the strategy. The steps for the optimal solution are as follows:

Step I Compute $C_{1}\left(N_{k}, a_{k}\right)$ the expected one step cost of adopting strategy $a_{k}$. This is the Hadamard product of $P_{a_{k}}$ and $C\left(N_{k}, a_{k}\right)$

Step II Compute the $\Pi_{a_{k}}$ the stationary transition matrix associated with strategy $a_{k}$. This is achieved by solving the following set of non-homogenous equations

$\Pi_{a_{k}}=\Pi_{a_{k}} P_{a_{k}} \quad$ subject to $\sum_{a_{k}} \Pi_{a_{k}}=1$

Step III Determine $V\left(N_{k}, a_{k}\right)$ the expected cost of the control strategy $a_{\mathrm{k}}$

$V\left(N_{k}, a_{k}\right)=\sum_{a_{k}} \Pi_{a_{k}} C\left(N_{k}, a_{k}\right)$

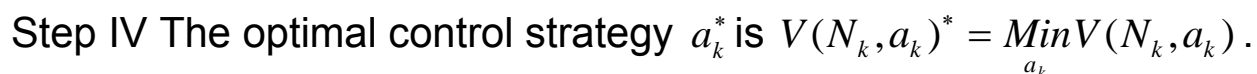

\section{ILLUSTRATIVE EXAMPLE}

We consider here a hypothetical three-grade Markovian manpower system with the following promotion control and associated cost matrices;

$$
\begin{array}{rlr}
P_{1} & =\left[\begin{array}{lll}
0.2 & 0.7 & 0.0 \\
0.0 & 0.1 & 0.8 \\
0.0 & 0.0 & 0.7
\end{array}\right] & C\left(P_{1}\right)=\left[\begin{array}{ccc}
3 & 20 & 0 \\
0 & 4 & 32 \\
0 & 0 & 34
\end{array}\right] \\
P_{2}=\left[\begin{array}{lll}
0.2 & 0.5 & 0.1 \\
0.0 & 0.5 & 0.4 \\
0.0 & 0.0 & 0.7
\end{array}\right] & C\left(P_{2}\right)=\left[\begin{array}{ccc}
4 & 14 & 10 \\
0 & 16 & 40 \\
0 & 0 & 54
\end{array}\right] \\
P_{3} & =\left[\begin{array}{ccc}
0.2 & 0.2 & 0.4 \\
0.3 & 0.33 & 0.2 \\
0.0 & 0.5 & 0.2
\end{array}\right] & C\left(P_{3}\right)=\left[\begin{array}{ccc}
18 & 25 & 29 \\
30 & 30 & 42 \\
0 & 20 & 24
\end{array}\right] \\
P_{4}= & {\left[\begin{array}{ccc}
0.0 & 0.3 & 0.4 \\
0.4 & 0.25 & 0.15 \\
0.0 & 0.4 & 0.35
\end{array}\right]} & C\left(P_{4}\right)=\left[\begin{array}{ccc}
0 & 18 & 28 \\
10 & 19 & 35 \\
0 & 28 & 40
\end{array}\right]
\end{array}
$$




$P_{5}=\left[\begin{array}{ccc}0.1 & 0.6 & 0.3 \\ 0.1 & 0.5 & 0.35 \\ 0.2 & 0.3 & 0.4\end{array}\right] \quad C\left(P_{5}\right)=\left[\begin{array}{ccc}10 & 20 & 20 \\ 14 & 40 & 40 \\ 20 & 35 & 60\end{array}\right]$

The following table summarises the stationary promotion control strategies and the expected total cost for the five different strategies

\begin{tabular}{|l|l|l|l|l|l|l|l|}
\hline Strategy & $C_{1}$ & $C_{2}$ & $C_{3}$ & $\pi_{1}$ & $\pi_{2}$ & $\pi_{3}$ & $V\left(N_{k}, a_{k}\right)$ \\
\hline 1 & 14.6 & 26 & 23.8 & 0 & 0 & 1 & 23.8 \\
\hline 2 & 8.8 & 24 & 31.5 & 0 & 0 & 1 & 31.5 \\
\hline 3 & 20.2 & 27.3 & 14.8 & $\frac{3}{37}$ & $\frac{8}{37}$ & $\frac{26}{37}$ & $\mathbf{1 7 . 9 4 1}$ \\
\hline 4 & 16.6 & 14 & 25.2 & $\frac{16}{119}$ & $\frac{40}{119}$ & $\frac{9}{17}$ & 20.279 \\
\hline 5 & 19 & 35.4 & 38.5 & $\frac{13}{61}$ & $\frac{24}{61}$ & $\frac{24}{61}$ & 47.784 \\
\hline
\end{tabular}

Hence the promotion control strategy with the minimum expected total cost is $P_{3}$

\section{CONCLUSION}

We have developed an optimal promotion control model for a Markovian manpower system. Using cost as an optimality performance criterion we were able to distinguish different promotion control strategies, that is showing that $V_{\pi^{*}}\left(N_{k}, a_{k}\right)$ is the optimal (minimum) value of $V_{\pi}\left(N_{k}, a_{k}\right)$. A dynamic programming technique was used to demonstrate the method of solution for an infinite horizon case. In the application $P_{3}$ gave the minimum value of 17.914 .

\section{REFERENCES}

Bartholonew, D. J., 1982. Stochastic Models for Social Processes; $3^{\text {rd }}$ ed. Wiley, Chichester.

Davies, G. S., 1982. Control of Grade Size in a partially Stochastic Markov Manpower Model. J. Appl. Prob. 19: 439 - 443.

Fry, M. J., Magazine, M. and Rao, U. S., 2003. Modeling Manpower Staffing in a fire Department; http://www.buzzle.com/editorial /9/2003-59662,asp

Gani, J., 1963. Formulae for Projecting Enrolment and Degrees Awarded in Universities; J. R. Stat. Soc. A. 126: 400- 409.

Kareem, B., 2005. A Review of Strategies for Manpower Planning in Depressed Economy; Proceedings of the International and Development Research Institute, Uyo.

MehImann, A., 1980. An Approach to Optimal Recruitment and Transition Strategies for Manpower Systems Using Dynamic Programming; J.Operat. Res.Soc. 31: 1009-1016

Nwaigwe, C.C., 2008. On the Recruitment and Promotion Manpower Control Using the Markov Theory. Journal of Research in Physical Sciences 4 (2): 87 - 91

Ossai, E. O., 2008. Statistical Control Models in Departmentalized and Graded Manpower System. Unpublished PhD Thesis, Department of Statistics, University of Nigeria Nsukka.

Uche, P. I., 1984: Some Control Measures for Markov Systems; Int. J. Math. Sci. Tech.; 15 (4): 479 - 483.

Young, A. and Almond, G., 1961. Predicting the Distribution of Staff; Comp. J. (3): 246-250 